\section{Romidepsin enhances the efficacy of cytarabine in vivo, revealing histone deacetylase inhibition as a promising therapeutic strategy for KMT2A-rearranged infant acute lymphoblastic leukemia}

Acute lymphoblastic leukemia (ALL) in infants diagnosed at less than 12 months of age is an aggressive malignancy with a poor prognosis. Rearrangements of the KMT2A gene (KMT2A-r) are present in up to $80 \%$ of cases, with 5 -year event-free survival (EFS) less than $40 \%{ }^{1}$ Dose intensive chemotherapy has been incorporated into contemporary treatment regimens; however, this has increased the burden of toxicity during therapy and late effects in survivors. ${ }^{1,2}$ There is a desperate need to identify novel therapies to improve outcome.

Histone deacetylase inhibition appears to be a promising therapeutic strategy for KMT2A-r infant ALL, with our recent chemo-genomic profiling identifying the potential for romidepsin. Romidepsin was shown to enhance the in vitro activity of cytarabine, a key component of infant ALL therapy, with an in vivo signal identified when combined with high-dose cytarabine. ${ }^{3}$ In this study, we investigate the in vivo synergy between romidepsin and cytarabine, determine the in vivo toxicity of this combination, and further explore the effect of romidepsin on the DNA damageresponse to cytarabine. All in vivo experiments were approved by the Animal Ethics Committee, Telethon Kids Institute, Perth, Australia.

To determine drug toxicity and efficacy upon completion of therapy, 7-week old female NOD/SCID mice were inoculated with $1 \times 10^{6}$ PER-785A cells. PER-785A is a genetically characterized cell line harboring the $t(4 ; 11)$ translocation. ${ }^{3}$ For all in vivo studies, drug treatment was commenced when the percentage of human $\mathrm{CD} 19^{+}$or $\mathrm{CD}_{4} 5^{+}$cells reached $1 \%$ in the bone marrow (BM), identified from extensive mapping of the leukemia cell kinetics for each model (Online Supplementary Figure S1). Treatment was scheduled to mimic the concepts of contemporary clinical trial design. Currently, novel agents are being investigated following induction therapy on the Interfant chemotherapy backbone, ${ }^{4}$ thus are introduced in the setting of low disease burden rather than at diagnosis where overt disease is evident in the BM. Mice were randomized into five groups of five mice and drug treatment was commenced on day 12 by intraperitoneal (i.p.) injection. Treatment groups comprised of vehicle control; $1.5 \mathrm{mg} / \mathrm{kg}$ romidepsin twice/week on Mondays and Thursdays; lowdose cytarabine $(5 \mathrm{mg} / \mathrm{kg}$ ) five times/week Monday to Friday; high-dose cytarabine $(100 \mathrm{mg} / \mathrm{kg})$ five times/week Monday to Friday; combination therapy with $1.5 \mathrm{mg} / \mathrm{kg}$ romidepsin and low-dose cytarabine. Treatment was administered for three weeks. Three days following completion of therapy mice were sacrificed and leukemia burden was determined by measuring the percentage of human $\mathrm{CD} 19^{+}$cells in the BM by flow cytometry with anti-human CD19-APC antibody. At the time of sacrifice, $0.2 \mathrm{~mL}$ of blood was obtained from each mouse by cardiac puncture and a complete blood count performed to determine the degree of myelosuppressive toxicity for each cohort. Single-agent activity was observed with the mean percentage of leukemic cells in the BM of $66.6 \%(P<0.01)$ for romidepsin and $27.3 \%(P<0.01)$ for low-dose cytarabine (Figure 1A). The reduction in leukemic burden was significantly enhanced with combination therapy with a reduction to a mean of $3.8 \%$ infiltration $(P<0.0001)$ (Figure 1A). Although treatment with high-dose cytarabine achieved clearance of leukemic cells from the BM, mice treated with high-dose cytarabine developed severe myelosuppression in comparison to the other cohorts (Figure 1B). In particular, there was a statistically significant reduction in mean hemoglobin (98 vs. $42.5 \mathrm{~g} / \mathrm{L}$; $P<0.0001)$, white blood cell $\left(2.43\right.$ vs. $0.13 \times 10^{9} / \mathrm{L}$; $P<0.0001)$ and platelet $\left(757\right.$ vs. $\left.294 \times 10^{9} / \mathrm{L} ; P<0.0021\right)$ count between the mice treated with romidepsin and lowdose cytarabine combination therapy compared to those treated with high-dose cytarabine.

Three xenograft models, PER-785, MLL-5 and MLL-14, were used to determine the response to drug treatment by EFS. MLL-5 and MLL-14 are well characterized patientderived xenografts which harbor $t(10 ; 11)$ and $t(11 ; 19)$ translocations respectively. ${ }^{5}$ MLL-5 and MLL-14 were selected to test whether findings could be validated in independent models with distinct translocation partners. For MLL-5, drug treatment commenced 11 days following injection of $1 \times 10^{6}$ cells, and for MLL-14, treatment commenced seven days following injection of $2 \times 10^{6}$ cells, corresponding to $1 \%$ of human $\mathrm{CD} 45^{+}$cells in the $\mathrm{BM}$. For each xenograft model, mice were randomized into four groups of eight mice prior to commencing therapy by i.p. injection. Given that the premise was to identify novel agents that would abrogate the toxicity of conventional chemotherapeutic agents, high-dose cytarabine was not further investigated. The rest of the treatment groups and schedules remained the same. Individual mouse EFS was calculated as the time in days from treatment initiation until mice reached a humane end point with evidence of leukemia-related morbidity. For MLL-5 and MLL-14, the anti-human CD45-PE antibody was used to determine the percentage of human CD $45^{+}$cells by flow cytometry. EFS curves were compared using log-rank test.

Compared to vehicle control, a significant survival benefit was seen with low-dose cytarabine alone for PER-785 and MLL-5 but not for MLL-14 (Figure 1C and Online Supplementary Table S1). This finding of differential sensitivity to cytarabine was validated in vitro (Online Supplementary Table S2). Our models thus provide representation of the known inter-patient variability in response to conventional chemotherapeutic agents. A significant survival advantage was not demonstrated for romidepsin monotherapy; however, combination therapy with romidepsin and low-dose cytarabine resulted in a profound and highly significant increase in median survival in all three xenograft models, compared to both vehicle control and low-dose cytarabine alone (Figure 1C and Online Supplementary Table S1). As expected, at the time the mice succumbed to leukemia, the disease burden was similar in all treatment groups (Online Supplementary Figure S2).

Next, we determined whether romidepsin enhanced the DNA-damage response of cytarabine in six of our previously characterized infant ALL cell lines. ${ }^{3}$ Phospho-H2A.X levels were determined by flow cytometry and western blotting (Online Supplementary Methods). For all cell lines, romidepsin was shown to significantly enhance the DNA double-strand break response of cytarabine (Figure 2A and $\mathrm{B}$ and Online Supplementary Figure S3). However, between cell lines there was variability in the extent to which DNAdamage was enhanced; this may reflect a potential for differences in the magnitude of clinical effect between patients. Romidepsin was not shown to increase the expression of other components of the DNA-damage signaling pathway (Online Supplementary Figure S4) or increase the inhibition of DNA repair (Online Supplementary Figure S5) when combined with cytarabine.

Using our panel of infant ALL cell lines, we subsequently determined whether romidepsin exhibited synergy with 
other conventional agents used in the Interfant chemotherapy backbone. In vitro synergy experiments were performed as previously described, with synergy scored using Chalice software, applying the Bliss-independence model. ${ }^{3}$ Synergy was demonstrated across all six cell lines for romidepsin combined with daunorubicin or 4hydroperoxycyclophosphamide (Table 1). Romidepsin was also shown to significantly enhance the DNA doublestrand break response of daunorubicin and 4-hydroperoxycyclophosphamide at the protein level (Figure 2C and D). Mixed responses across the cell line panel were observed when romidepsin was combined with vincristine (synergy in four cell lines) or dexamethasone (synergy in three cell lines), whereas antagonism was observed in four out of six cell lines when romidepsin was combined with methotrexate

or

L-asparaginase (Table 1). Our data provide support for romidepsin to be scheduled prior to or during cytarabineand cyclophosphamide-based therapy within the current standard Interfant chemotherapy backbone, namely protocol IB and the second half of OCTADAD.

There is a desperate need to identify novel agents for integration into up-front therapy to improve the dismal outcome of infants with KMT2A-r ALL. The KMT2A-fusion protein has altered histone-methyltransferase activity with characteristic changes to chromatin and transcriptome. ${ }^{6}$ Several components of the KMT2Acomplex can be targeted by novel agents. However, in order to integrate any novel drug into infant therapy protocols, the drug needs to be available in a preparation suitable for administration to infants, i.e. as an oral suspension or formulated for intravenous use. Furthermore, current infant ALL protocols contain up to ten different chemotherapeutics, given in an intricate drug delivery scheme over 24 months. Evidence is required as to where in the standard chemotherapy backbone novel drugs can be safely administered with maximal effect. Simultaneous administration with a conventional chemotherapeutic
A

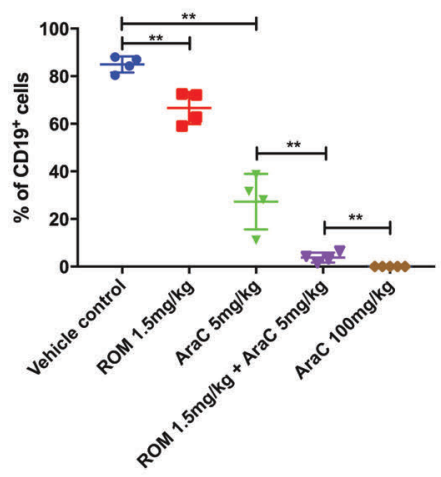

B

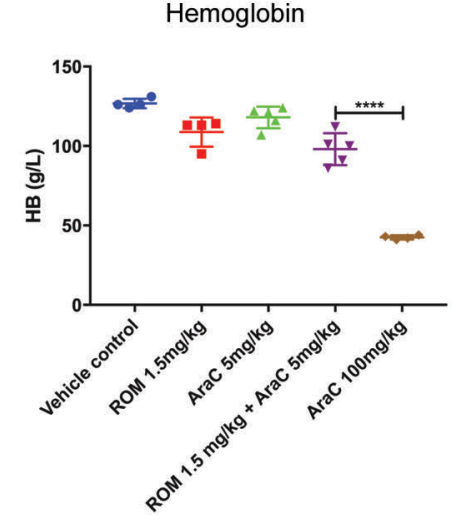

C

PER-785

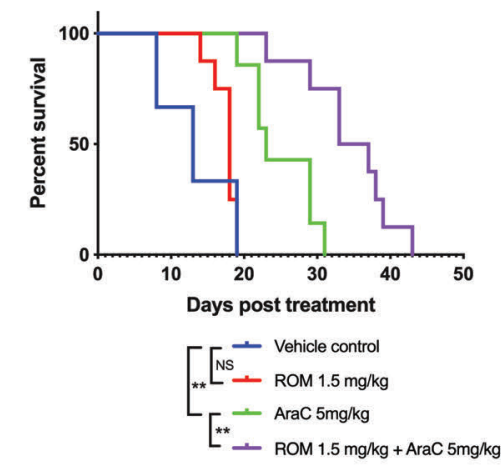

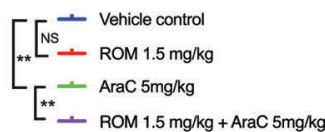

Figure 1. Romidepsin and low-dose cytarabine combination therapy reduces leukemia burden and improves survival in mice. (A and B) Mice injected with PER-785 leukemia cells were treated with vehicle control, $1.5 \mathrm{mg} / \mathrm{kg}$ romidepsin (ROM) twice weekly, low-dose (5 $\mathrm{mg} / \mathrm{kg}$ ) cytarabine (AraC) five times a week, high-dose (100 mg/kg) AraC five times a week, or a combination of $1.5 \mathrm{mg} / \mathrm{kg}$ ROM and low-dose AraC for three weeks. Mice were sacrificed three days following completion of therapy ( $n=4-5$ mice/group). (A) Leukemia burden in the bone marrow and (B) Complete blood count measurements. (C) Kaplan-Meier survival curves of mice injected with PER-785, MLL-5, or MLL-14 leukemia cells that were treated with vehicle control, $1.5 \mathrm{mg} / \mathrm{kg}$ ROM, $5 \mathrm{mg} / \mathrm{kg}$ AraC, or combination of $1.5 \mathrm{mg} / \mathrm{kg}$ ROM and $5 \mathrm{mg} / \mathrm{kg}$ AraC for three weeks ( $n=7-8$ mice/group). Two-tailed unpaired Student $t$-test was used to analyze leukemia burden and complete blood count measurements, and log-rank test for Kaplan-Meier survival analysis. Error bars represent mean \pm Standard Deviation. $* * P<0.01 ; * * * P<0.001 ; * * * * P<0.0001$.
White blood cells

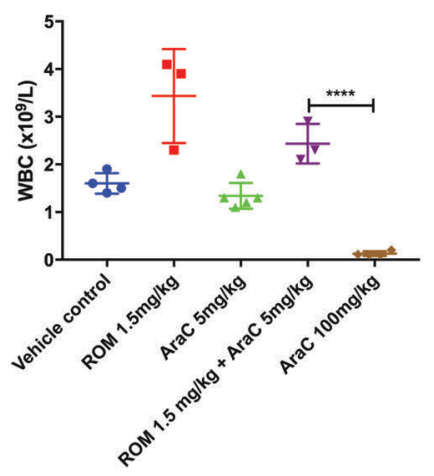

MLL-5

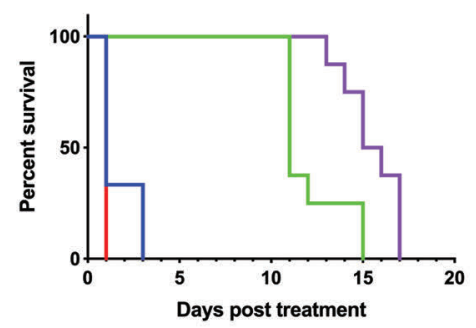

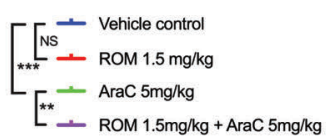

Platelets

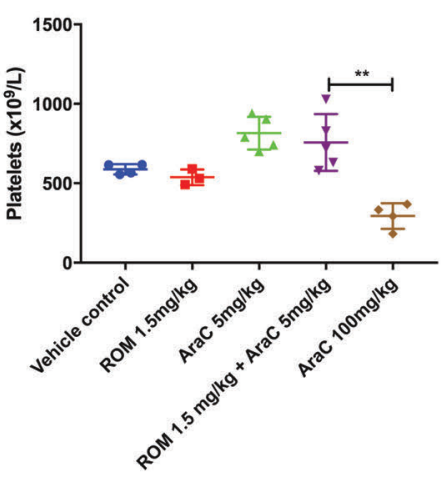

MLL-14

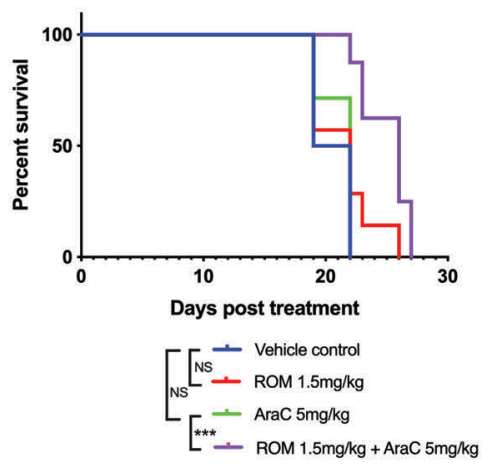


A

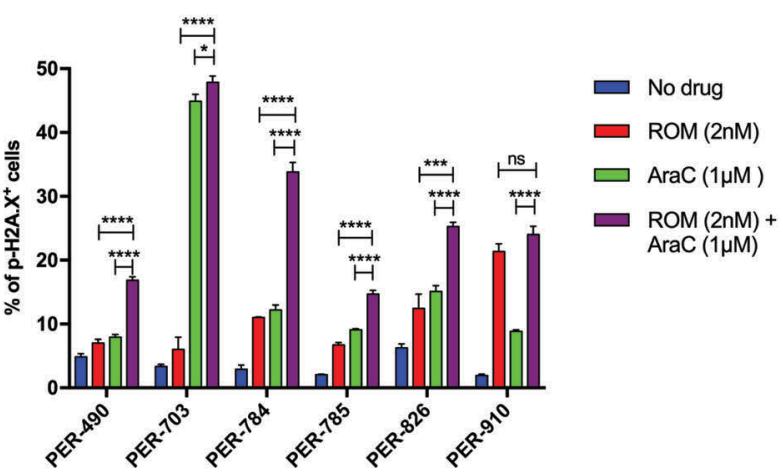

B
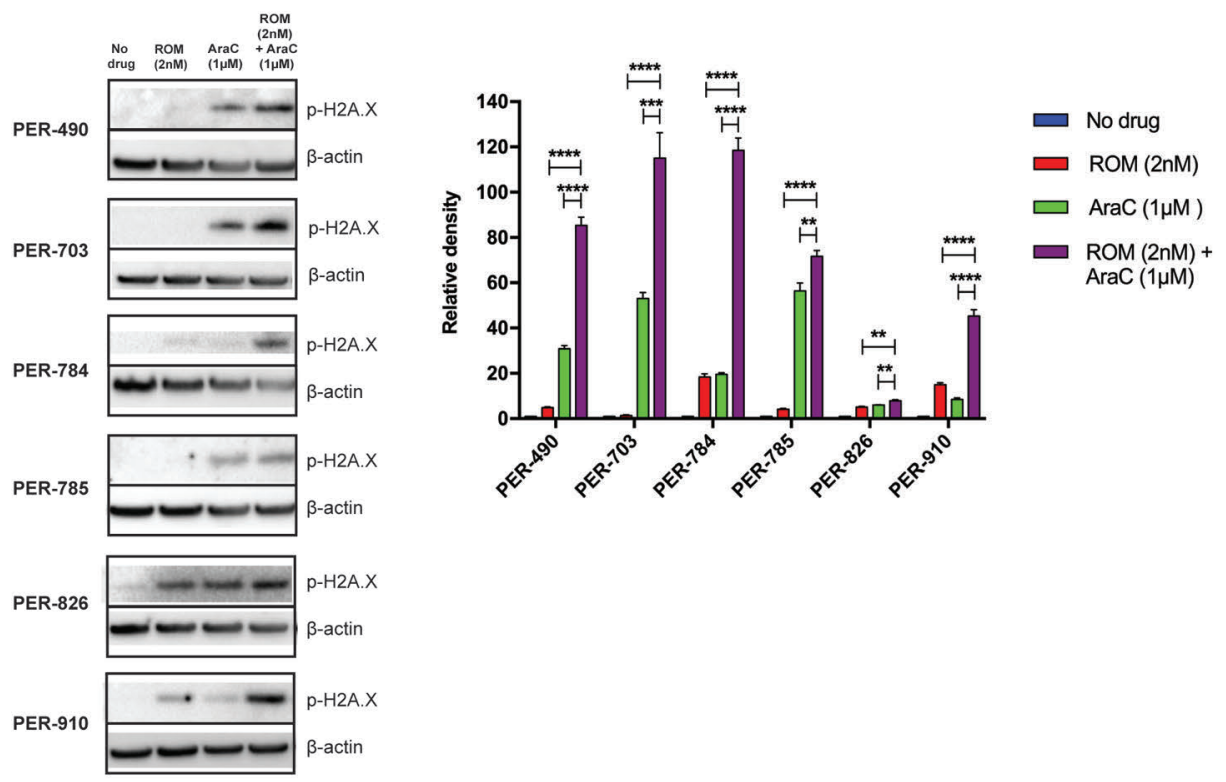

C

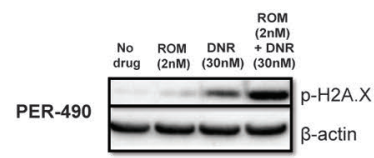

D
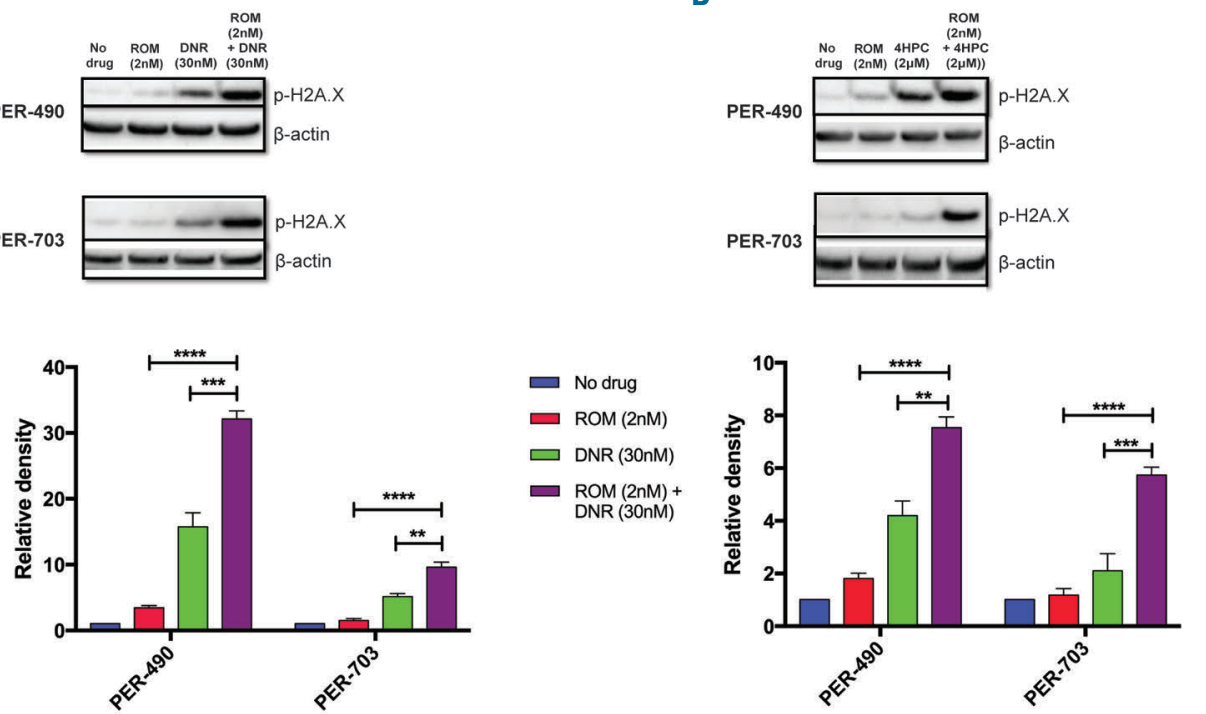

- Nodrug

$\square$ ROM (2nM)

$\square 4 \mathrm{HPC}(2 \mu \mathrm{M})$

- $\operatorname{ROM}(2 \mathrm{nM})+$ $4 \mathrm{HPC}(2 \mu \mathrm{M})$

Figure 2. Romidepsin enhances the DNA double-strand break response of DNA damaging chemotherapeutic agents. (A and B) Leukemia cell lines were treated with $2 \mathrm{nM}$ romidepsin (ROM), $1 \mu \mathrm{M}$ cytarabine (AraC), or combination of $2 \mathrm{nM}$ ROM and $1 \mu \mathrm{M}$ AraC for 24 hours (h). (A) Cells were stained with PI and phosphoH2A.X and analyzed by flow cytometry. (B) Western blot analysis of phospho-H2A.X protein levels in the treated cell lines (left). Bar graphs depict phospho$\mathrm{H} 2 \mathrm{~A} . \mathrm{X} / \beta$-actin densitometry ratios that were normalized to ratios calculated for non-treated controls (right). (C) Leukemia cell lines were treated with $2 \mathrm{nM}$ ROM, $30 \mathrm{nM}$ Daunorubicin (DNR), or combination of $2 \mathrm{nM}$ ROM and $30 \mathrm{nM}$ DNR for $24 \mathrm{~h}$. (D) Leukemia cell lines were treated with $2 \mathrm{nM}$ ROM, $2 \mu \mathrm{M}$ 4-hydroperoxycyclophosphamide (4HPC), or combination of $2 \mathrm{nM} \mathrm{ROM}$ and $2 \mu \mathrm{M} 4 \mathrm{HPC}$ for $24 \mathrm{~h}$. (C and D) Western blot analysis of phospho-H2A.X protein levels in the treated cell lines (top). Bar graphs depict phospho-H2A.X/ $\beta$-actin densitometry ratios that were normalized to ratios calculated for non-treated controls (bottom). Data were collected from three independent experiments and analyzed using the two-tailed unpaired Student $t$-test. Error bars represent mean \pm Standard Deviation. $* P<0.05 ; * * P<0.01 ; * * * P<0.001 ; * * * * P<0.0001$. 
Table 1. Total and maximum in vitro synergy scores between romidepsin and conventional chemotherapy agents.

Total Synergy

\begin{tabular}{|l|c|c|c|c|c|c|}
\cline { 2 - 7 } \multicolumn{1}{c|}{} & PER-785 & PER-826 & PER-490 & PER-703 & PER-784 & PER-910 \\
\hline Romidepsin - 4HPC & 163.81 & 208.53 & 405.84 & 554.97 & 11.75 & 407.3 \\
\hline Romidepsin - Vincristine & -45.17 & 126.12 & 197.64 & -124.51 & 232.8 & 258.05 \\
\hline Romidepsin - Daunorubicin & 262.49 & 453.39 & 246.16 & 358.8 & 467.32 & 216.58 \\
\hline Romidepsin - Dexamethasone & -216.78 & 333.72 & -237.55 & -652.41 & 137.69 & 481.94 \\
\hline Romidepsin - Cytarabine & 468.97 & 378.79 & 417.57 & 118.46 & 225.93 & 777.06 \\
\hline Romidepsin - Methotrexate & 163.86 & -52.53 & 96.74 & -204.69 & -152.69 & -20.63 \\
\hline Romidepsin - L-Asparaginase & -218 & -65.9 & 313 & -262 & -31.7 & 136 \\
\hline
\end{tabular}

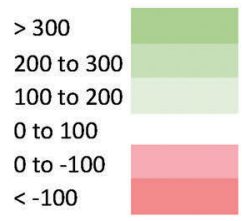

\begin{tabular}{|c|c|c|c|c|c|c|}
\hline & PER-785 & PER-826 & PER-490 & PER-703 & PER-784 & PER-910 \\
\hline Romidepsin - 4HPC & 14.43 & 14.14 & 23.51 & 25.19 & 12.07 & 25.46 \\
\hline Romidepsin - Vincristine & 4.45 & 10.64 & 14.36 & 4.77 & 18.74 & 19.96 \\
\hline Romidepsin - Daunorubicin & 16.42 & 27.85 & 16.15 & 13.76 & 21.33 & 23.96 \\
\hline Romidepsin - Dexamethasone & 4.52 & 22.5 & 0.36 & 3.15 & 8.1 & 25.42 \\
\hline Romidepsin - Cytarabine & 30.88 & 20.94 & 28.54 & 8.53 & 12.71 & 35.83 \\
\hline Romidepsin-Methotrexate & 15.64 & 3.41 & 20.02 & 2.76 & 0.22 & 11.29 \\
\hline Romidepsin - L-Asparaginase & 9 & 2 & 14 & 5 & 9 & 11 \\
\hline
\end{tabular}

agent would only be considered for clinical use if the drug combination achieves more than each drug individually, with a tolerable toxicity profile, and if antagonism can be excluded. Our study focused on KMT2A-r infant ALL; however, use of romidepsin is not specific for this indication, with current extensive clinical investigation in combination regimens for several adult hematologic malignancies, including anthracycline based-therapies, ICE (ifosfamide, carboplatin and etoposide) and gemcitabine-containing regimens. ${ }^{7}$ Our study provides strong evidence that romidepsin can be combined to augment the effect of the conventional chemotherapeutic agent, cytarabine, in infants with KMT2A-r ALL without undue myelosuppression, which is one of the predominant dose-limiting side effects of chemotherapy. The potential for histone deacetylase inhibition in ALL has also been highlighted in several recent studies, ${ }^{8-10}$ including strong in vivo efficacy of panobinostat against human KMT2A-r ALL. ${ }^{8}$ Taken together, there is mounting evidence to support the use of histone deacetylase inhibitors in the next generation of clinical trials for infants with KMT2A-r ALL.

Laurence C. Cheung, ${ }^{1,2}$ Mark N. Cruickshank, ${ }^{1}$ Anastasia M. Hughes, ${ }^{1}$ Sajla Singh,, Grace-Alyssa Chua, ${ }^{1}$ Jette Ford, ', Emanuela Ferrari, Joyce Oommen, ${ }^{1}$ Sébastien Malinge, ${ }^{1}$ Richard B. Lock, ${ }^{3}$ Ursula R. Kees' and Rishi S. Kotecha $a^{1,2,4,5}$

'Division of Children's Leukaemia and Cancer Research, Telethon Kids Cancer Centre, Telethon Kids Institute, University of Western Australia, Perth; ${ }^{2}$ School of Pharmacy and Biomedical Sciences, Curtin University, Perth; ${ }^{3}$ Children's Cancer Institute, Lowy Cancer Research Centre, UNSW, Sydney; ${ }^{4}$ Department of Haematology and Oncology, Perth Children's Hospital, Perth and 'Division of Paediatrics, School of Medicine, University of Western Australia, Perth, Australia

Funding: this work was supported by project grants from the Children's Leukaemia and Cancer Research Foundation, Western Australia; the Telethon-Perth Children's Hospital Research Fund, Western Australia; and The Royal Australasian College of Physicians (RACP)/The Kids Cancer Project Research Establishment Fellowship. MNC is supported by the Woolworths Children's Leukaemia and Cancer Research Foundation Fellowship. SS is supported by The Kids' Cancer Project, New South Wales. RBL (NHMRC
APP1059804) and RSK (NHMRC-APP1142627) are supported by Fellowships from the National Health and Medical Research Council of Australia.

Correspondence: RISHI S. KOTECHA.

rishi.kotecha@health.wa.gov.au

doi:10.3324/haematol.2018.192906

Information on authorship, contributions, and financial \& other disclosures was provided by the authors and is available with the online version of this article at www. haematologica.org.

\section{References}

1. Kotecha RS, Gottardo NG, Kees UR, Cole CH. The evolution of clinical trials for infant acute lymphoblastic leukemia. Blood Cancer J. 2014;4:e200.

2. Gandemer V, Bonneau J, Oudin C, et al. Late effects in survivors of infantile acute leukemia: a study of the L.E.A program. Blood Cancer J. 2017;7(1):e518.

3. Cruickshank MN, Ford J, Cheung LC, et al. Systematic chemical and molecular profiling of MLL-rearranged infant acute lymphoblastic leukemia reveals efficacy of romidepsin. Leukemia. 2017;31(1):4050 .

4. Pieters R, Schrappe M, De Lorenzo P, et al. A treatment protocol for infants younger than 1 year with acute lymphoblastic leukaemia (Interfant-99): an observational study and a multicentre randomised trial. Lancet. 2007;370(9583):240-250.

5. Richmond J, Carol H, Evans K, et al. Effective targeting of the P53MDM2 axis in preclinical models of infant MLL-rearranged acute lymphoblastic leukemia. Clin Cancer Res. 2015;21(6):1395-1405.

6. Winters AC, Bernt KM. MLL-Rearranged Leukemias-An Update on Science and Clinical Approaches. Front Pediatr. 2017;5:4.

7. Petrich A, Nabhan C. Use of class I histone deacetylase inhibitor romidepsin in combination regimens. Leuk Lymphoma. 2016;57(8):1755-1765.

8. Garrido Castro P, van Roon EHJ, Pinhancos SS, et al. The HDAC inhibitor panobinostat (LBH589) exerts in vivo anti-leukaemic activity against MLL-rearranged acute lymphoblastic leukaemia and involves the RNF20/RNF40/WAC-H2B ubiquitination axis. Leukemia. 2018;32(2):323-331.

9. Savino AM, Sarno J, Trentin L, et al. The histone deacetylase inhibitor givinostat (ITF2357) exhibits potent anti-tumor activity against CRLF2-rearranged BCP-ALL. Leukemia. 2017;31(11):23652375.

10. Waibel M, Vervoort SJ, Kong IY, et al. Epigenetic targeting of Notch1-driven transcription using the HDACi panobinostat is a potential therapy against T-cell acute lymphoblastic leukemia. Leukemia. 2018;32(1):237-241. 